\title{
Interaction between FGF and BMP signaling pathways regulates development of metanephric mesenchyme
}

\author{
Andrew T. Dudley, ${ }^{1,2}$ Robert E. Godin, ${ }^{1}$ and Elizabeth J. Robertson ${ }^{3}$ \\ Department of Molecular and Cellular Biology, Harvard University, The Biological Laboratories, \\ Cambridge, Massachusetts 02138 USA
}

\begin{abstract}
Nephrogenesis in the mouse kidney begins at embryonic day 11 and ends $\sim 10$ days postpartum. During this period, new nephrons are continually being generated from a stem-cell population-the nephrogenic mesenchyme-in response to signals emanating from the tips of the branching ureter. Relatively little is known about the mechanism by which the nephrogenic mesenchyme cell population is maintained at the tips of the ureter in the presence of signals promoting tubulogenesis. Previous studies have shown that a loss of $B m p 7$ function leads to kidney defects that are a likely result of progressive loss of nephrogenic mesenchyme by apoptosis. The studies presented here demonstrate that BMP7 signaling can prevent apoptosis in explants of metanephric mesenchyme. The surviving mesenchyme cell population, however, is not competent to respond to signals promoting tubulogenesis. In conjunction with FGF2, BMP7 promotes growth and maintains competence of the mesenchyme in vitro. In addition, FGF2 and BMP7 signaling, both independently and in combination, inhibit tubulogenesis. Interestingly, FGF2 and BMP7 also promote expansion of the stromal progenitor cell population in whole kidney culture. Because BMP7 is not produced by stromal progenitor cells, these data suggest a novel interaction between the nephrogenic mesenchyme and stromal progenitor cell populations. A model for the regulation of nephrogenesis by FGF and BMP signaling is presented.
\end{abstract}

[Key Words: Kidney; fibroblast growth factor; bone morphogenetic protein-7; stromal progenitor cell; apoptosis; competence]

Received January 5, 1999; revised version accepted April 27, 1999.

Development of the metanephric kidney depends on a program of reciprocal inductive interactions between two mesodermal derivatives, the ureteric bud and the nephrogenic mesenchyme (for review, see Saxen 1987). During the eleventh day postcoitum $(\mathrm{dpc})$, the ureteric bud, an outgrowth of the Wolffian duct, contacts the metanephric blastema. Following contact, inductive interactions establish two cell fates within the blastemathe stromal progenitor cells and the nephrogenic mesenchyme (Aufderheide et al. 1987). Signals from the ureteric bud promote survival of the nephrogenic mesenchyme and initiate in this cell population a process leading to tubule formation. Concomitantly, signals provided by the mesenchyme stimulate growth and branching of the ureteric bud that ultimately forms the collecting duct system. Continued development of the metanephros is dependent on coordination of these two processes.

\footnotetext{
${ }^{1}$ These authors contributed equally to this work.

${ }^{2}$ Present address: Department of Genetics, Harvard Medical School, Boston, Massachusetts 02115 USA.

${ }^{3}$ Corresponding author.

E-MAIL ejrobert@fas.harvard.edu; FAX (617) 496-6770.
}

Little is known about the signals involved in maintaining a population of nephrogenic mesenchyme at the tips of the branching ureter. Preserving this cell population conceptually requires three signaling events. First, the tendency of metanephric mesenchyme to undergo apoptosis must be blocked (Weller et al. 1991; Koseki et al. 1992; Coles et al. 1993). Second, because branching of the ureter and formation of nephrons subdivides and depletes the nephrogenic mesenchyme cell population, self renewal via cell proliferation is necessary (Sainio et al. 1994). Third, a signal is required to oppose tubulogenesis promoted by the ureter, thereby reserving a subset of cells for future rounds of tubulogenesis. It is likely that at least some of these signals are downstream of $E m \times 2$, a transcription factor expressed in the ureter (Miyamoto et al. 1997). In Emx2 null mutant embryos, the ureter contacts the blastema but fails to promote survival or proliferation of the mesenchyme. In part, this may be attributable to failure of the mutant ureter to induce robust expression of certain genes, such as the transcription factors Wilms' tumor gene-1 (WT1) and Pax2, in the adjacent metanephric mesenchyme (Dressler et al. 1990; Kreidberg et al. 1993). Analysis of null mutants demonstrated that WT1 function is required cell autonomously 
for response of the mesenchyme to signals emanating from the ureter (Kreidberg et al. 1993). Genes downstream of Emx2 and WT1 may include receptor tyrosine kinases (RTK) and their respective ligands, such as members of the fibroblast growth factor (FGF) gene family. One member of the family, FGF2/bFGF, prevents apoptosis and promotes expression of WT1 in cultures of isolated rat metanephric mesenchyme (Perantoni et al. 1995a). Following treatment, these explants remain competent to undergo tubulogenesis in response to signals from spinal cord (Barasch et al. 1997). It is likely that FGF family members have several roles during metanephric development, as their receptors (FGFRs) are expressed in most cell populations in the kidney (Orr-Urtreger et al. 1991; Stark et al. 1991).

Another gene likely to have a role in maintaining the nephrogenic mesenchyme is bone morphogenetic protein-7 (Bmp 7). Expression of Bmp7 in the mesenchyme is initiated when the ureter contacts the metanephric blastema and is maintained in the mesenchyme throughout kidney development (Dudley et al. 1995; Lyons et al. 1995; Dudley and Robertson 1997; Godin et al. 1998). A loss-of-function mutation in $B m p 7$ does not affect the initial inductive interactions between the ureter and metanephric blastema (Dudley et al. 1995; Luo et al. 1995). Approximately $48 \mathrm{hr}$ after the first induction, however, a progressive cessation of nephrogenesis is observed. The onset of the phenotype is coincident with observed apoptosis in the cortical region (Luo et al. 1995; Dudley and Robertson 1997). By 16.5 dpc, the mutant kidneys are small, lack condensed mesenchyme, and contain many cystic structures. Together, these data suggest that BMP7 signaling is required for the survival of metanephric mesenchyme in vivo.

The studies presented here provide new insights into the role of $B m p 7$ in the maintenance of nephrogenic mesenchyme. We show that purified recombinant human BMP7 can promote the survival of mesenchyme cells in the absence of the ureter. Unlike in cultures containing freshly isolated metanephric mesenchyme, however, treated mesenchyme does not undergo tubulogenesis when co-cultured with spinal cord. In contrast, mesenchyme treated with FGF2 displays a weak tubulogenic response to signals from spinal cord. In combination, BMP7 signaling potentiates the response of mesenchyme to FGF signaling, enabling the formation of many tubules in cultured mesenchyme following addition of spinal cord. These data suggest that synergy between FGF2 and BMP7 signaling maintains the nephrogenic mesenchyme cell population. In addition, signaling through these two pathways can repress differentiation of mesenchyme in whole kidney cultures in a reversible manner, an effect that correlates with expansion of the stromal progenitor cell population. Collectively, these data suggest that signaling within the nephrogenic mesenchyme and between the nephrogenic mesenchyme and stromal progenitor cell population is necessary for maintenance of nephrogenic mesenchyme at the tips of the branching ureter during kidney development.

\section{Results \\ Bmp7 promotes survival but not differentiation of metanephric mesenchyme}

Previous analysis of null mutants demonstrated that loss of $B m p 7$ function results in the progressive cessation of nephrogenesis, which is correlated with the appearance of apoptotic cells in the nephrogenic zone (Dudley et al. 1995; Luo et al. 1995). In vivo, mutant kidneys develop fewer nephrons than wild-type, however the nephrons that form are functional. These data suggest that $B m p 7$ function has a role in survival of metanephric mesenchyme but does not affect its ability to undergo tubulogenesis. This interpretation is in contrast to that presented by Vukicevic et al. (1996). These authors demonstrated that mesenchyme explant cultures treated with $50 \mathrm{ng} / \mathrm{ml}$ BMP7 express the transcription factor Pax2 and the extracellular matrix molecule laminin A chain, markers of induced mesenchyme and renal epithelium. These data suggest that BMP7 signaling is necessary and sufficient for induction of metanephric mesenchyme. In our attempts to replicate their results, however, we did not observe induction of mesenchyme adjacent to BMP7coated beads (Godin et al. 1998).

To address these differences we re-examined the effect of recombinant BMP7 on isolated metanephric mesenchyme in vitro. As per Vukicevic et al. (1996), BMP7 was added directly to the culture medium. Explants were cultured for 24-96 hr with medium changes daily or every other day. Some of the experiments were performed under serum-free conditions, although the data presented here were derived from cultures exposed to $10 \%$ fetal bovine serum (FBS). Results were essentially the same regardless of variations in culture conditions. After $48 \mathrm{hr}$ in culture, control explants treated with bovine serum albumin (BSA) displayed significant degeneration (data not shown). Co-culture with spinal cord resulted in induction of mesenchyme and subsequent tubulogenesis (Fig. 1A) as demonstrated by strong expression of the transcription factor Pax 2 and the secreted signaling molecule Wnt4 (Fig. 1C,E; Dressler et al. 1990; Stark et al. 1994). In contrast, $50 \mathrm{ng} / \mathrm{ml} \mathrm{BMP7}$ promotes survival but not aggregation of the mesenchyme cells (Fig. 1B) or expression of Pax2 and Wnt4 (Fig. 1D,F). Therefore, BMP7 cannot mimic the growth and differentiation effects of spinal cord on metanephric mesenchyme. Similar results were obtained in explants cultured for 48-96 hr in the presence of $10-1000 \mathrm{ng} / \mathrm{ml}$ of BMP7. In addition, we tested whether $B m p 7$ function was required for induction of mesenchyme. Embryos from $B m p 7^{\text {lac } Z}$ heterozygous intercrosses were obtained at $11.5 \mathrm{dpc}$. Spinal cord derived from wild-type mice and metanephric mesenchyme isolated from these $B m p 7^{l a c Z}$ embryos were recombined and grown under serum-free conditions for 72 hr. Wild-type or heterozygous mesenchyme underwent tubulogenesis when recombined with like spinal cord (Fig. 1G). Similarly, Bmp7 mutant spinal cord can promote tubulogenesis in Bmp7 mutant mesenchyme (Fig. $1 \mathrm{H})$. In contrast to wild-type cultures, mutant mesenchyme explants remained small and appeared to contain 

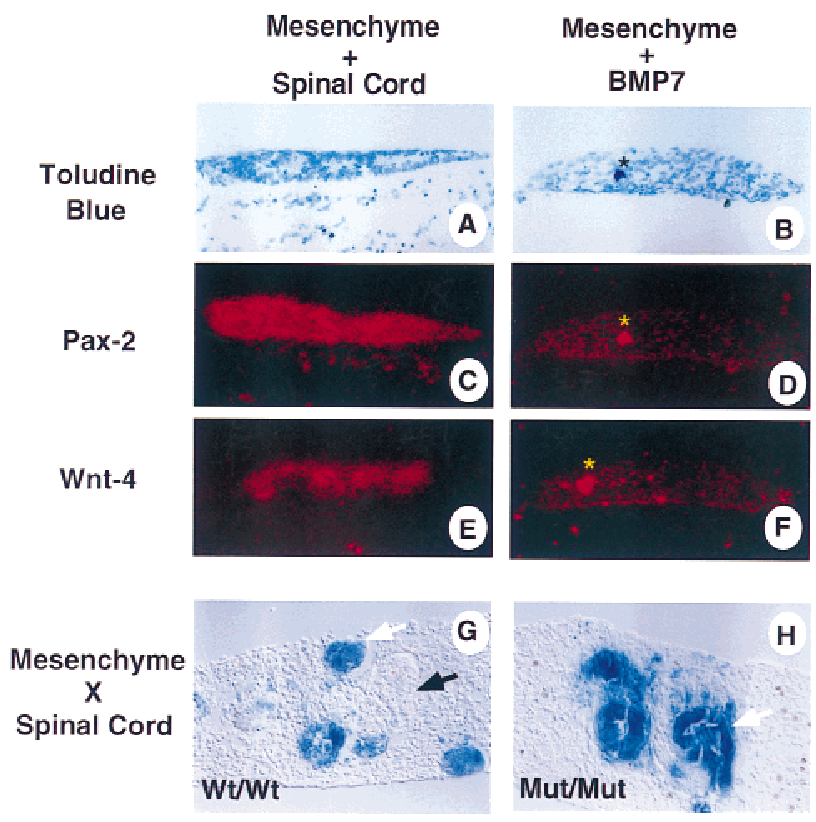

Figure 1. BMP7 signaling does not induce nephrogenic mesenchyme. Mesenchyme was isolated from 11.5-dpc embryos and grown in culture for $48 \mathrm{hr}$ in the presence of spinal cord $(A, C, E)$ or recombinant BMP7 protein $(B, D, F)$. After culture, explants were sectioned and processed for in situ hybridization. $(A, B)$ Bright-field and $(C-F)$ dark-field exposures of serial sections. In the presence of spinal cord the mesenchyme condenses, forms immature epithelium, and displays strong expression of $\mathrm{Pax} 2$ $(C)$ and Wnt4 $(E)$. In contrast, mesenchyme grown in the presence of BMP7 remains loose and does not express Pax2 $(D)$ or Wnt4 $(F)$. The asterisk in panels $B, D$, and $F$ indicate an artifact. Recombinant cultures of wild-type spinal cord and $B m p 7^{1 a c Z} /+$ mesenchyme display many LacZ ${ }^{+}$early tubules (white arrow) as well as nonstaining, more mature tubules ( $G$; black arrow). Cultures of $B m p 7$ mutant mesenchyme isolated from $B m p 7^{1 a c Z}$ em bryos recombined with mutant spinal cord also display well formed tubules $(H)$.

fewer tubules. Therefore, mutant tissue in recombinant culture develops similar to mutant tissue in vivo (Dudley et al. 1995; Luo et al. 1995). Together, these data demonstrate that BMP7 signaling is neither sufficient nor required for induction of nephrogenic mesenchyme or subsequent tubulogenesis.

Although BMP7 does not promote differentiation of the mesenchyme, it appeared from these studies and Godin et al. (1998) that BMP signaling prevents cell death in mesenchyme explants. To directly test this possibility, explants were cultured in the presence of a bead coated with BSA or BMP7 for $48 \mathrm{hr}$ then incubated in medium containing TOPRO-1, a fluorescent vital dye. As shown previously, a translucent zone appears in the mesenchyme adjacent to beads soaked in BMP7 but not BSA (Fig. 2A,C; Godin et al. 1998). Here we demonstrate that apoptotic cells are distributed uniformly in control explants whereas fluorescent label is restricted from cells adjacent to BMP7-coated beads (Fig. 2B,D). We compared survival of mesenchyme treated with BMP7-coated beads to that of mesenchyme treated with FGF2-coated beads. Previous data described a role for FGF2 as a survival factor for metanephric mesenchyme (Perantoni et al. 1995a; Godin et al. 1998). In these cultures we find an overall reduction of TOPRO-1 staining throughout the mesenchyme explant consistent with FGF2 acting as a potent survival factor (Fig. 2E,F). Interestingly, FGF2treated cultures appear as more coherent cell masses than control or BMP7-treated explants. The limited region of surviving cells surrounding BMP7-coated beads may be attributable to differences in protein release from heparin-Sepharose (FGF2) and Affi-gel blue (BMP7) beads or may be indicative of short-range signaling by BMP7. Isolated mesenchyme incubated in medium containing BMP7 with daily medium changes, however, also did not survive as well as FGF2-treated cultures (data not shown). Collectively, these data demonstrate that although both FGF2 and BMP7 signaling can rescue metanephric mesenchyme from cell death, FGF2 may block apoptosis more effectively. To determine which cell populations are rescued from apoptosis through either
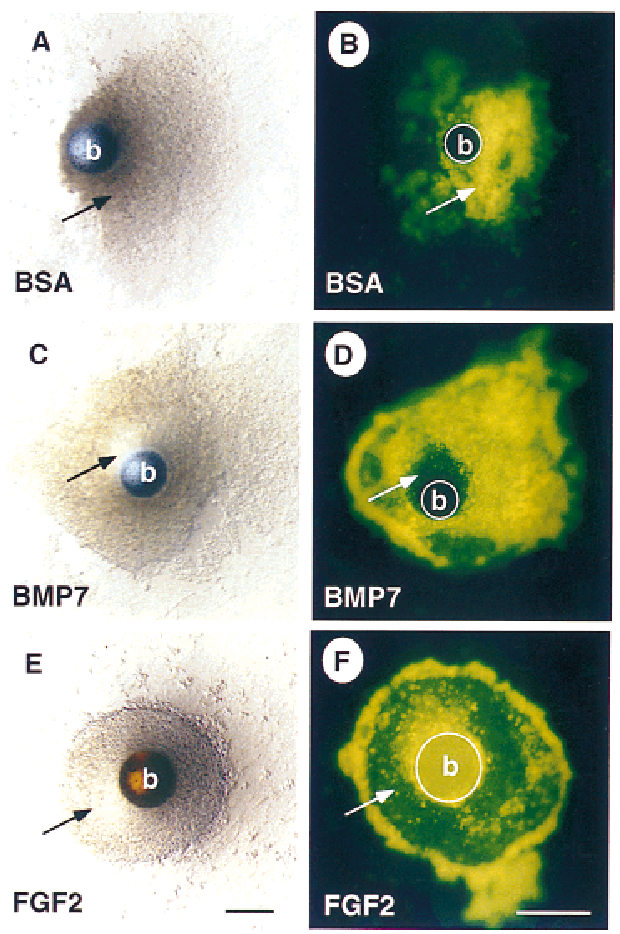

Figure 2. BMP7 promotes survival of the nephrogenic mesenchyme. Mesenchyme was isolated from 11.5-dpc embryos and grown in culture for $48 \mathrm{hr}$ in the presence of a bead containing BSA $(A, B)$, BMP7 $(C, D)$, or FGF2 $(E, F)$. Explants treated with BSA appear granular and refractory to light. In contrast, there is a well organized, translucent region of mesenchyme in the vicinity of the BMP7-soaked bead (cf. cells indicated by arrow in $A$ and $C$ ). Staining with the vital dye TOPRO-1 reveals that cells in the BSA-treated mesenchyme are dying as indicated by fluorescent signal $(B)$, whereas, in the BMP7-treated mesenchyme, cells in the translucent region do not fluoresce, indicating that these cells are viable (cf. cells indicated by arrow in $B$ and $D$ ). Interestingly, most of the cells in the explant survive when treated with FGF2 $(E, F)$. Note that the heparin-Sepharose beads in $E$ and $F$ display autofluorescence. (b) Bead. Scale bar, $100 \mu \mathrm{m}$. 
Dudley et al.

BMP7 or FGF2 signaling, we performed whole-mount in situ hybridization on explants cultured with beads (data not shown). In brief, control and treated cultures displayed uniform, low-level expression of WT1, a marker of nephrogenic mesenchyme cells; $B F 2$, a winged-helix transcription factor expressed exclusively by stromal progenitor cells; and lacked expression of Pax2. These data suggest that the surviving cell population is comprised of a mixture of stromal progenitor cells and uninduced nephrogenic mesenchyme cells.

\section{Bmp7 and FGF2 signaling function synergistically in metanephric mesenchyme}

One caveat to mesenchyme explant experiments is that added factors must first promote survival of the mesenchyme if any effect of the factors on differentiation is to be ascertained. Because BMP7 does not provide robust protection from apoptosis, we concluded that previous experiments using isolated metanephric mesenchyme were unlikely to reveal the function of $B m p 7$. To overcome this problem, we tested the activity of BMP7 in combination with FGF2. For our experiments, mesenchyme was obtained from $B m p 7^{l a c Z}$ heterozygous embryos so that induced mesenchyme and the resultant tubules could be identified readily by LacZ activity (Godin et al. 1998). Mesenchyme was cultured in the presence of factors for 48-96 hr with medium changes daily. Following incubation, cultures were fixed and stained for LacZ activity. Explants cultured in medium without additional factors degenerated (data not shown). Cultures treated with $50 \mathrm{ng} / \mathrm{ml} \mathrm{BMP7}$ remained small and degenerated slowly (Fig. 3A). A few scattered LacZ ${ }^{+}$cells are occasionally detected in these cultures. As reported previously, FGF2 treatment promoted growth and survival of the culture (Fig. 3B). Many LacZ ${ }^{+}$cells were present in these cultures, however, these cells did not form aggregates as observed in induced mesenchyme (Fig. 1G; Godin et al. 1998). Explants treated with both BMP7 and FGF2 were consistently larger than explants cultured with FGF2 alone, and they rarely contain $\mathrm{LacZ}^{+}$cells (Fig. 3C). The absence of significant LacZ activity in these cultures suggests that none of these treatments promotes induction of the mesenchyme (Godin et al. 1998). Accordingly, neither Pax2 nor Wnt4 transcripts were detected in these cultures (Fig. 3E,F,H,I) but were readily detected in mesenchyme explants co-cultured with spinal cord (Fig. 1C,E). Therefore, BMP7 signaling promotes growth but not differentiation of the mesenchyme in the presence of FGF2 signaling.

Because $B m p 7$, a marker of induced metanephric mesenchyme, was expressed in explants treated with FGF2 but not those exposed to both FGF2 and BMP7, we wanted to test whether nephrogenic mesenchyme cells were also present in these cultures. Therefore, we tested whether treated mesenchymes could form nephrons in response to signaling by spinal cord. Metanephric mesenchyme was cultured for $48 \mathrm{hr}$ as described above. Explants were then transferred to medium lacking these factors and two pieces of freshly dissected spinal cord

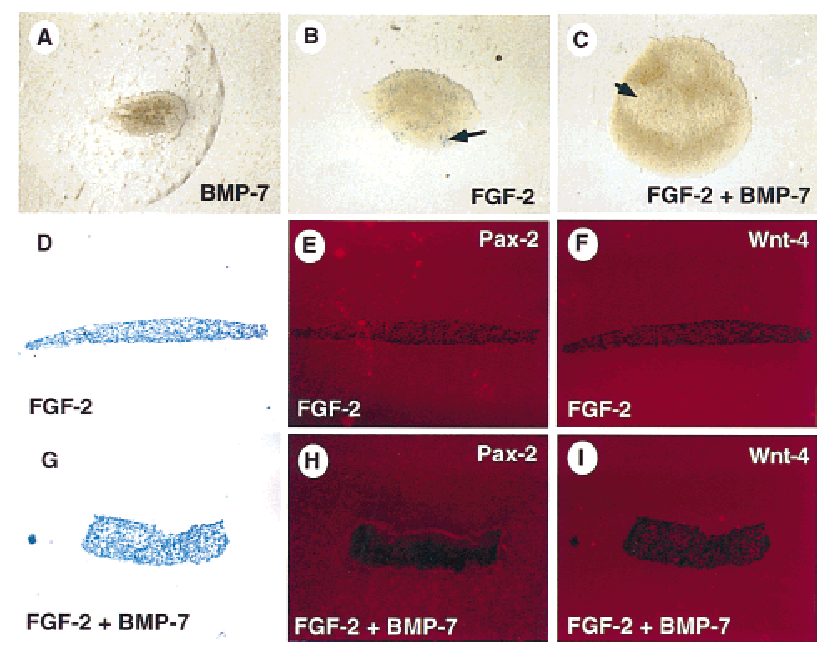

Figure 3. BMP7 and FGF2 function synergistically to promote survival and growth of mesenchyme explants. Mesenchyme was isolated from 11.5-dpc $B m p 7^{l a c Z} /+$ embryos and grown in culture for $48 \mathrm{hr}$ in the presence of BMP7 $(A)$, FGF2 $(B, D-F)$, or both BMP7 and FGF2 $(C, G-I)$. After culture, explants were stained in X-gal or fixed and processed for in situ hybridization. Mesenchyme treated with BMP7 displays limited survival. FGF2 promotes better survival of the mesenchyme. Treatment with both factors results in survival and growth of the isolated mesenchyme. Interestingly, these cultures display translucent regions $(C$, arrow $)$ similar to that observed around BMP7- or FGF2-soaked beads. Marker analysis reveals LacZ ${ }^{+}$cells in cultures treated with FGF2 ( $B$, arrow). In addition, neither FGF2 $(D-F)$ or FGF2 + BMP7- $(G-I)$ treated cultures express Pax2 or Wnt4, demonstrating that induction has not occurred in these explants.

were placed in contact with the mesenchyme. After an additional 48-hr incubation, cultures were fixed and stained for LacZ activity. In the absence of added factors, control cultures degenerated during the experiment and did not respond to signals from spinal cord /data not shown). Explants pre-incubated in the presence of 100 $\mathrm{ng} / \mathrm{ml}$ of FGF2 displayed many single $\mathrm{LacZ}^{+}$mesenchymal cells and a few small aggregates following induction by spinal cord (Fig. 4A). Similarly, mesenchyme pretreated with $50 \mathrm{ng} / \mathrm{ml} \mathrm{BMP7}$ responded only weakly to inductive signals (Fig. 4B). The combination of $50 \mathrm{ng} / \mathrm{ml}$ of BMP7 and $100 \mathrm{ng} / \mathrm{ml}$ of FGF2, however, resulted in a robust response to spinal cord so that tubule formation was clearly observed (Fig. 4C, E) and was qualitatively similar to freshly isolated mesenchyme co-cultured with spinal cord for $48 \mathrm{hr}$ (Fig. 4D,F). Increased doses of BMP7 or FGF2 did not appreciably alter the response of mesenchyme to spinal cord (data not shown). The above experiment was also performed using lithium rather than spinal cord as an inducer of nephrogenic mesenchyme (Davies and Garrod 1995; Godin et al. 1998; data not shown). In these experiments, pretreatment of the mesenchyme with both FGF2 and BMP7 resulted in large regions of the lithium-induced explant displaying LacZ activity, whereas FGF2 alone yielded only a few LacZ ${ }^{+}$ cells and BMP7-treated explants were mostly negative. 


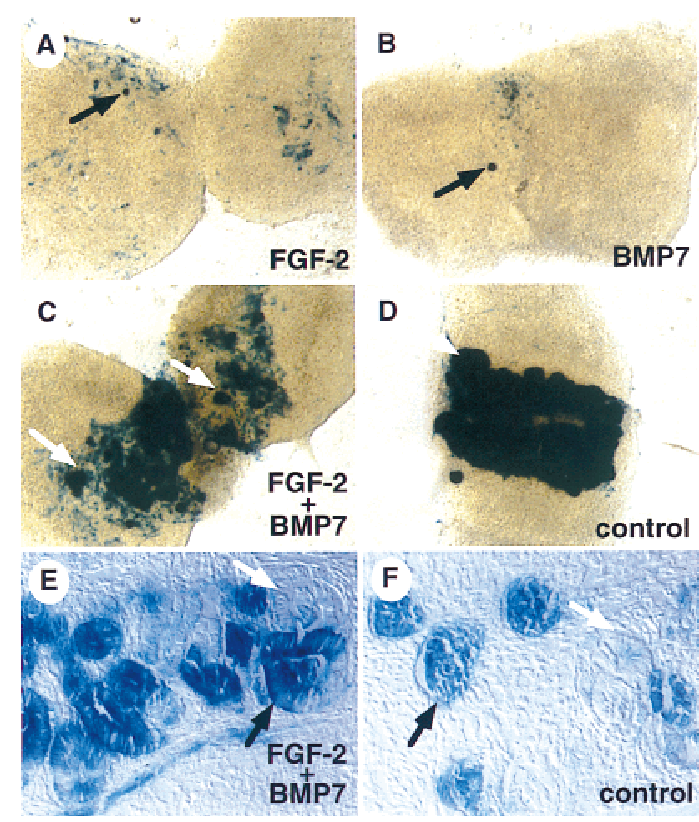

Figure 4. BMP7 and FGF2 signaling maintain nephrogenic mesenchyme in vitro. Mesenchyme was isolated from 11.5-dpc $B m p 7^{1 a c Z} /+$ embryos and grown in culture for $48 \mathrm{hr}$ in the presence of recombinant FGF2 $(A)$, BMP7 $(B)$, or both $(C)$. Mesenchyme was then placed in contact with spinal cord and grown for an additional $48 \mathrm{hr}$. As a control, freshly isolated mesenchyme was placed immediately in contact with spinal cord $(D)$. After culture, explants were stained in X-gal. Neither FGF2 nor BMP7 alone maintain a population of cells competent to undergo induction, though a few $\mathrm{LacZ}^{+}$cells and small aggregates of cells are often seen in these cultures (black arrows). In contrast, pretreatment with both factors allows a robust response of the explant to induction (white arrows). Sections through FGF2 + BMP7 $(E)$ and control $(F)$ cultures demonstrate that stained regions contain tubules and forming glomeruli (black arrows) as well as more mature, nonstaining tubules (white arrows). In addition, regions of stained, nonaggregated cells are readily observed in cultures pretreated with FGF2 + BMP7.

As expected, tubules did not form in these cultures as lithium can only mimic the initial inductive event (Davies and Garrod 1995; Godin et al. 1998). These data strongly suggest that BMP7 signaling potentiates the activity of FGF2, resulting in the maintenance of nephrogenic mesenchyme cells capable of responding to inductive signals.

\section{BMP7 and FGF2 signaling oppose tubulogenesis}

To address whether BMP7 and FGF2 act synergistically to maintain a population of nephrogenic mesenchyme in the context of a developing kidney, we examined the effect of these factors on whole organ cultures. Metanephric rudiments from 11.5-dpc embryos derived from heterozygous intercrosses were cultured on Nuclepore membrane rafts in medium containing $10 \%$ FBS. After 48-96 $\mathrm{hr}$ in culture, the rudiments were fixed in 100\% methanol. To visualize ureteric branching and nephron formation, explant cultures were immunostained for laminin, a basement membrane protein in epithelial tissues. Under these culture conditions, the ureter in wildtype kidneys branches several times and many nephrons are formed (Fig. 5A). In contrast, the ureter in mutant kidneys branches less and the resultant explant is significantly smaller than that from wild-type littermates (Fig. 5B). In addition, mutant explants are packed with large, abnormally shaped nephrons (Fig. 5, cf. D and E). This is surprising because we predicted that apoptosis of nephrogenic mesenchyme cells in mutant kidneys would result in the absence of tubules at the termini of the ureteric branches. Instead, this observation suggests that in the absence of BMP7 signaling, the nephrogenic mesenchyme also undergoes premature differentiation.

To test whether BMP7 activity can antagonize signals promoting tubulogenesis, we cultured wild-type kidneys in the presence of BMP7, FGF2, or both for $48 \mathrm{hr}$ and then determined the extent of tubulogenesis following staining for laminin. After $48 \mathrm{hr}$, nephrons are found adjacent to most of the termini of the ureteric branches (Fig. 5A,D). In kidneys treated with $50 \mathrm{ng} / \mathrm{ml}$ BMP7, the ureter branches normally and many tubules are observed (Fig. 5C). Unlike the situation in control explants, however, a significant portion of the ureteric termini remained surrounded by condensed mesenchyme and lack mature nephrons (Fig. 5F). At higher doses, BMP7 can significantly inhibit tubulogenesis (data not shown). Likewise, $100 \mathrm{ng} / \mathrm{ml}$ FGF2 decreases formation of nephrons in the periphery (Fig. 5G,J). In combination, these two molecules strongly inhibit nephrogenesis, resulting in the majority of ureteric tips lacking nephrons (Fig. $5 \mathrm{H}, \mathrm{K})$. To determine whether the inhibition of nephrogenesis is caused by an adverse effect of this treatment regimen on the peripheral mesenchyme population, some kidney explants were transferred to medium without added factors following the initial culture period and incubated for an additional $48 \mathrm{hr}$. In most cases, removal of BMP7 and FGF2 from the medium permitted tubulogenesis to resume in the periphery, resulting in nephrons adjacent to the tips of the ureter (Fig. 5, cf. $\mathrm{H}$ and $\mathrm{K}$ with I and L). Additionally, in some treated explants the peripheral mesenchyme population was labeled with the fluorescent lineage tracing dye DiI. Following transfer of the explants to medium without added factors, DiI-labeled cells were incorporated mainly into the stromal cells surrounding the nephrons, further demonstrating that these cells remain viable and functional following treatment with BMP7 and FGF2 (data not shown). The ability of BMP7 signaling to inhibit nephron formation was corroborated using a second assay. Mesenchyme explants containing a bead soaked in BSA or BMP7-containing media were co-cultured with spinal cord for 24$48 \mathrm{hr}$. Following serial sectioning, nephrogenesis was analyzed in these cultures both histologically and by expression of Pax2 and Wnt4. Condensed mesenchyme and immature tubules were often detected adjacent to beads soaked in BSA but rarely within 100-150 $\mu \mathrm{m}$ of BMP7coated beads (data not shown). Collectively, these data provide strong evidence that BMP7 activity can repress nephrogenesis in induced metanephric mesenchyme. 
Dudley et al.

Figure 5. BMP7 and FGF2 signaling oppose tubulogenesis. Whole kidneys were isolated at 11.5-dpc and grown on Nuclepore filter rafts. After culture, explants were stained with an anti-laminin antibody via indirect immunofluorescence. Low magnification $(A-C$, $G-I)$ and high magnification $(D-F, J-L)$ views of explants are presented. $(A, D)$ Kidney explant grown for $48 \mathrm{hr}$ in the presence of $10 \%$ FBS. $(B, E) B m p 7$ mutant kidney grown for 48 $\mathrm{hr}$ in the presence of $10 \%$ FBS. Arrows in $D$ and $E$ indicate nephrons that have formed in the periphery. Note how mutant tubules appear larger and less organized than wild-type. Wildtype kidneys grown in the presence of recombinant BMP7 $(C, F)$, FGF2 $(G, J)$, or both factors $(H, K)$. BMP7 seems to have little effect on the branching of the ureter, however, tips of the ureter are often found free of nephrons $(F$, arrow). FGF2 signaling alone or in combination with BMP7 also reduces nephron formation in the periphery of the explant (arrows in $J$ and $K)$. In contrast to kidneys treated with BMP7 alone, however, FGF2 signaling causes a rind of cells to form in the periphery of the explant $(G$, arrow). In combination, both factors promote an even greater expansion of this outer cell population $(H$, arrow). Explants treated with FGF2 + BMP7 for $48 \mathrm{hr}$, subsequently placed in fresh culture media lacking these factors and grown for an additional $48 \mathrm{hr}$, display extensive tubulogenesis in the periphery of the kidney $(I, L)$.
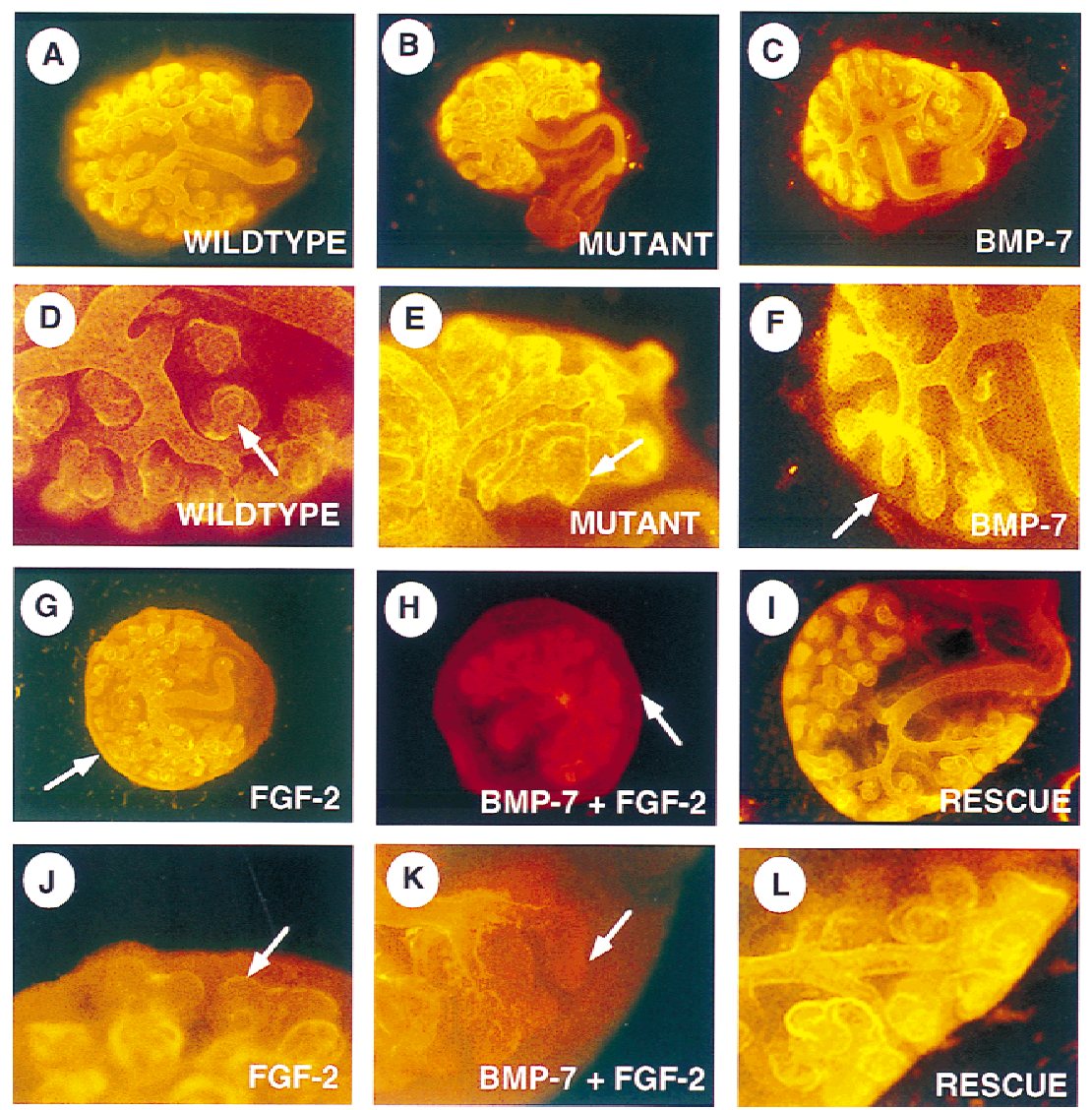

Interestingly, in addition to preventing tubulogenesis, BMP7 and FGF2 together promote overgrowth of a peripheral mesenchyme cell population (Fig. 5, cf. A, C, and $\mathrm{G}$ with $\mathrm{H}$ ). This region contains at least two cell types - the nephrogenic mesenchyme, precursor cells of nephrons, and progenitor cells of the kidney stroma. Results from DiI labeling (described above) suggest that the expanded cell population is composed predominantly of stromal progenitor cells. To determine which cell populations is affected by treatment with these factors, we examined the expression of marker genes by wholemount in situ hybridization following explant culture. Nephrogenic mesenchyme cells were identified by expression of the transcription factors WT1 and Pax2, and the secreted signaling molecule Bmp7. Pax2 and Bmp7 are also expressed strongly in the ureteric epithelium. In control kidney cultures, expression of these markers is observed in mesenchyme cells adjacent to the tips of the ureter (Fig. 6A,C,E). Similar expression patterns are observed in kidneys exposed to both BMP7 and FGF2 (Fig. $6 \mathrm{~B}, \mathrm{D}, \mathrm{F})$. Interestingly, none of these markers are expressed in the peripheral mesenchyme in treated kidneys. In contrast, the more peripheral population of mesenchyme was positive for expression of $B F 2$, a wingedhelix transcription factor that marks the stromal progenitor cell population (Fig. 6G,H; Hatini et al. 1996). Sections through these explants localize BF2 expression to the peripheral stromal progenitor cell population (Fig.
6I,J). Therefore, together, BMP7 and FGF signaling promote expansion of the stromal progenitor cell population while repressing differentiation of the adjacent nephrogenic mesenchyme.

\section{FGFRs are expressed in the nephrogenic region}

Because exogenous sources of BMP7 and FGF2 activity have strong effects on the metanephric mesenchyme-cell populations in explant and whole kidney cultures, we next asked whether the spatial and temporal expression patterns of the relevant genes are consistent with their proposed roles. We and others have demonstrated by both in situ hybridization and analysis of embryos carrying the $B m p 7^{l a c Z}$ allele that $B m p 7$ is expressed in both the ureteric epithelium and the adjacent condensed mesenchyme, but not in the surrounding stromal progenitor cells (Dudley et al. 1995; Lyons et al. 1995; Dudley and Robertson 1997; Godin et al. 1998). It has been shown that Fgf2 is expressed in the ureter of the rat (Perantoni et al. 1991; Perantoni et al. 1995a,b; Cancilla et al. 1996). We have been unable to confirm this expression pattern in the mouse by in situ hybridization but have demonstrated expression of Fgf2 in kidneys from 12.5-dpc embryos by RT-PCR (data not shown). It is unlikely, however, that FGF2 is the relevant signal as Fgf2 null mutant mice do not display kidney defects (Zhou et al. 1998). Rather than examine the expression patterns of all 18 


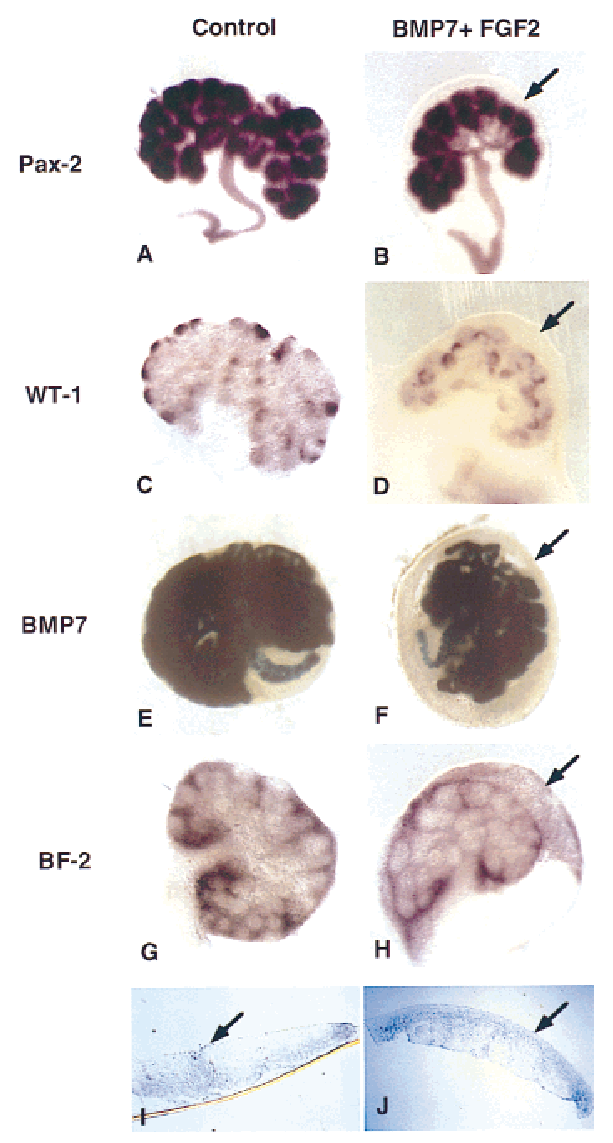

Figure 6. BMP7 and FGF2 promote expansion of the stromal progenitor cell population. Whole kidneys were isolated at 11.5 dpc and grown for $48 \mathrm{hr}$ in culture containing $10 \%$ FBS in the absence $(A, C, E, G, I)$ or in the presence of both BMP7 and FGF2 $(B, D, F, H, J)$. After culture, explants were processed for wholemount in situ hybridization or stained in X-gal. (A) Pax2 is expressed in the ureter and in the condensed mesenchyme. (B) A similar expression pattern is observed in the treated kidneys. WT1 is expressed in the condensed mesenchyme associated with the tips of the branching ureter in both control $(C)$ and treated kidneys $(D)$. X-gal staining for the $B m p 7^{\text {lac } Z}$ allele also shows a similar expression pattern in both the control $(E)$ and treated $(F)$ explants. In all cases the expanded rind of cells in the periphery of the kidney do not express any of these markers of induced mesenchyme (arrow). The stromal precursor marker $B F 2(G)$, however, is expressed in this expanded population of cells $(H)$. Sections through $G$ and $H$ reveal that $B F 2$ is expressed throughout the expanded region of mesenchyme cells (arrow, $I, J)$. The apparent BF2 signal in the nephrogenic mesenchyme is attributable to the layer of stromal progenitor cells that encompasses the entire explant $(G, H)$.

members of the FGF family, we decided to focus on the expression of the two FGFRs, expressed in mesenchyme populations in the developing kidney (Orr-Urtreger et al. 1991). As described previously, Fgfr1 is expressed in the mesenchyme but not in the ureter of wild-type kidneys (Fig. 7A). Surprisingly, in cultured kidneys treated with BMP7 and FGF2, Fgfr1 expression is detected only in a peripheral rind of mesenchyme (Fig. 7B). As this cell population expresses BF2-but not WT1, Bmp7, or Pax2
(Fig. 6) - it is likely that Fgfr1 is expressed by stromal progenitor cells but not by condensed mesenchyme. It appears, however, that Fgfr1 may be expressed in only a subpopulation of stromal progenitor cells because expression is detected only at the periphery of the explant, not in the BF2-positive cells adjacent to the condensed mesenchyme. In contrast, in both untreated and treated whole kidney cultures, Fgfr2 is expressed in the ureter and condensed mesenchyme but not in the stromal progenitor cell population (Fig. 7C,D). Therefore, it appears that the nephrogenic mesenchyme and stromal progenitor cells use different receptors to transduce FGF signals.

\section{Discussion}

Previous studies demonstrated that BMP7 signaling is required for development of the murine kidney (Dudley et al. 1995; Luo et al. 1995). These reports suggested that BMP7 produced by the metanephric mesenchyme promotes cell survival via an autocrine pathway. There is an important caveat to this interpretation. Survival and differentiation of the metanephric mesenchyme is dependent on interaction with the ureter. Therefore a decrease in growth or function in a $B m p 7$-deficient ureter could adversely affect survival of the mesenchyme. To overcome this problem, we employed two experimental approaches. First, we analyzed the effect of recombinant BMP7 on isolated metanephric mesenchyme cultured in the absence of ureteric epithelium. Second, we examined the effect of BMP7 on spinal cord induced differentiation of mesenchyme. Using these methods and others we

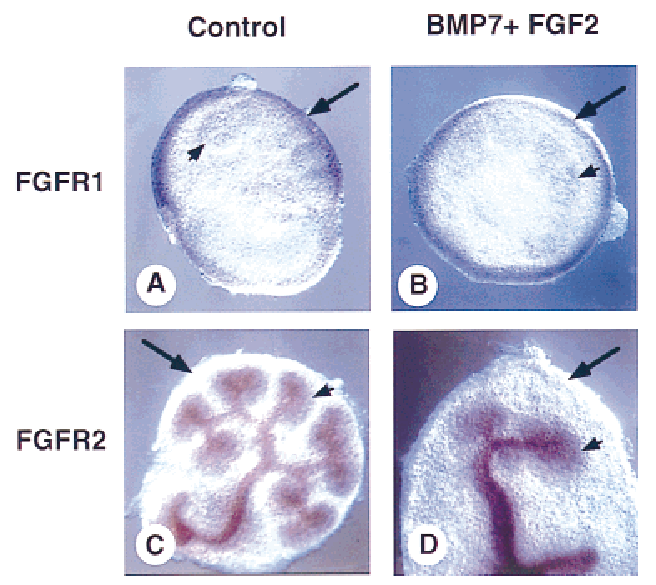

Figure 7. FGFR expression in BMP7/FGF2-treated kidney explants. Whole kidneys were isolated at $11.5 \mathrm{dpc}$ and grown for $48 \mathrm{hr}$ in culture in $10 \%$ FBS $(A, C)$ or in the presence of BMP7 and FGF2 $(B, D)$. After culture, explants were processed for whole-mount in situ hybridization. Fgfr1 is expressed in the most peripheral mesenchyme of both control $(A)$ and treated $(B)$ explants. Fgfr2 is expressed in the ureteric tissue as well as in the condensed mesenchyme of both control $(C)$ and treated $(D)$ explants but is absent from the peripheral mesenchyme. (long arrow) Stromal cells; (short arrow) condensed nephrogenic mesenchyme. 
demonstrate that synergy between BMP7 and FGF2 signal transduction regulates several key processes during nephrogenesis.

\section{BMP7 and FGF2 are not inducers}

of metanephric mesenchyme

Analysis of null mutations in $B m p 7$ by two groups resulted in controversy over whether BMP7 signaling mediates induction of metanephric mesenchyme (Luo et al. 1995) or acts to promote survival of the nephrogenic mesenchyme (Dudley et al. 1995; Dudley and Robertson 1997). Using in vitro methods, Vukecevic et al. (1996) provided support for a role of BMP7 in induction of mesenchyme. These investigators demonstrated that explants of metanephric mesenchyme express the epithelial cell markers Pax2 and laminin A chain when cultured for $48 \mathrm{hr}$ in the presence of $50 \mathrm{ng} / \mathrm{ml} \mathrm{BMP7}$. Despite numerous attempts, we were unable to replicate these data. Under a variety of culture conditions, doses of BMP7 ranging from $10-1000 \mathrm{ng} / \mathrm{ml}$ did not result in the formation of condensates or the expression of Pax2 and Wnt4 in cultures of isolated mesenchyme. These data demonstrate that BMP7 signaling alone is not sufficient to promote induction of mesenchyme explants but these experiments do not rule out a role for $B m p 7$ in the process of induction. In support of a role for BMP7 signaling in induction of metanephric mesenchyme, previous experiments demonstrated that antisense oligonucleotides directed against $B m p 7$ transcripts inhibit spinal cord-induced differentiation of mesenchyme (Vukicevic et al. 1996). Here we show that $B m p 7$ mutant mesenchyme can be induced by mutant spinal cord in serum-free culture, making it likely that the inhibition of tubulogenesis observed in the previous study is not attributable specifically to loss of BMP7 production in either the mesenchyme or spinal cord. These data, combined with our previous results (Dudley et al. 1995; Godin et al. 1998), conclusively demonstrate that BMP7 signaling is neither sufficient nor required for induction of metanephric mesenchyme.

In addition, we find no evidence to support the notion that FGF2 signaling mediates early inductive events in explants of mouse metanephric mesenchyme, as suggested for the rat (Perantoni et al. 1995a). We detect weak expression of some markers of induction (i.e. Bmp7 and WT1) following treatment of isolated mesenchyme with FGF2, but the level of expression is generally well below that of mesenchyme induced by spinal cord or lithium chloride (this report; Godin et al. 1998). Although these low levels of expression might represent a weak inductive response, the fact that these markers can be detected in untreated cultures up to $24 \mathrm{hr}$ after explanting (A.T. Dudley and R.E. Godin, unpubl.) suggests that FGF2 signaling acts to maintain cells in an induced state in the absence of continuous inductive signals.

If explants treated with BMP7 or FGF2 are not induced, how do we account for the presence of $\mathrm{LacZ}^{+}$cells in similarly treated cultures (Fig. 3; Godin et al. 1998)? Perdurance of LacZ activity in the absence of continued gene expression is unlikely as $\mathrm{LacZ}^{+}$cells are not detected in mesenchyme cultured for the same amount of time in the presence of both FGF2 and BMP7. In addition, we demonstrated previously that LacZ activity is rapidly lost in maturing tubules (Godin et al. 1998). Our previous results suggest that $B m p 7$ is an early marker of induced nephrogenic mesenchyme cells that is expressed before or independently of Pax2 and Wnt4 (Godin et al. 1998). Therefore, we suggest that Bmp7 expression marks one of the earliest stages of induction of nephrogenic mesenchyme cells. Because they can exist as single, $\mathrm{LacZ}^{+}$mesenchymal cells in these explants (Fig. 3 and 4), it is likely that the phenotype is maintained, in part, through autocrine signaling that is not BMP7-dependent (Godin et al. 1998). FGF2 signaling promotes survival of these cells and, in addition, may help maintain this phenotype. On the other hand, BMP7 signaling alone or in combination with FGF2 signaling greatly reduces or eliminates $\mathrm{LacZ}^{+}$cells in these cultures (Fig. 3). It is unlikely that these cells die or change fate, as tubules form following induction by spinal cord in cultures of mesenchyme pretreated with BMP7 and FGF2 (Fig. 4). One possible explanation for this observation is that BMP7 signaling negatively regulates $B m p 7$ transcription either directly or through the inhibition of another signaling pathway. Alternatively, increased BMP7 signaling may force cells into an earlier developmental state characterized by the absence of expression of any currently known gene associated with induction.

\section{Synergy between BMP7 and FGF2 signaling regulates nephrogenesis}

The data presented here demonstrate three roles for BMP7 signaling during development of the metanephros. First, they demonstrate that BMP7 activity can preserve nephrogenic mesenchyme cells in vitro in the absence of continuous induction. The mechanism underlying the maintenance of this cell population may include the prevention of cell death, the stimulation of cell proliferation, or the preservation of competence to respond to inducing factors. The data presented here (Fig. 2), in combination with our previous results (Godin et al. 1998), provide conclusive evidence that BMP7 signaling promotes survival of mesenchyme cells. In addition, we demonstrate that FGF2 prevents apoptosis of metanephric mesenchyme isolated from the mouse as shown previously for the rat (Perantoni et al. 1991; Perantoni et al. 1995a). One interesting question is why, if both BMP7 and FGF2 signaling prevent apoptosis in mesenchyme explants (Fig. 2), can only explants treated with FGF2 be induced (Fig. 4)? There are several possible explanations. FGF2 signaling may be more effective for promoting survival of nephrogenic mesenchyme cells as suggested in Figure 2. Alternatively, FGF2 may promote proliferation of the nephrogenic stem cells in addition to ensuring their survival. In this case, FGF2 signaling might increase the number of nephrogenic mesenchyme cells 
past a critical level necessary for tubulogenesis. This nonlinear relationship between cell number and biological effect has been termed the 'community effect' (Gurdon 1988). Moreover, previous studies suggest that cell mass may be a critical factor during nephron formation (Gossens and Unsworth 1972). In addition, it is possible that FGF2, but not BMP7, regulates competence of nephrogenic mesenchyme cells to respond to inducing signals. This possibility is consistent with the observation that induced, LacZ ${ }^{+}$cells are rarely detected following induction by spinal cord in explants treated with BMP7 alone but are readily observed in cultures treated with FGF2 (Fig. 4). Finally, BMP7 signaling alone may alter the fate of nephrogenic mesenchyme cells. It has been suggested that nephrogenic mesenchyme cells and stromal progenitor cells are derived from a common progenitor cell. Perhaps BMP7 signaling directs cells to the stromal cell lineage in the absence of FGF signaling. A role for BMP signaling in cell fate determination has been described in cultures of neural crest cells (Varley et al. 1995; Shah et al. 1996). In order to examine the above possibilities, it is important to be able to distinguish cells derived from the nephrogenic mesenchyme from those of the stromal lineage in explant culture in the absence of induction. Unfortunately, all markers described to date for the nephrogenic mesenchyme are dependent on inductive signals.

A second, unexpected, role for BMP7 was revealed by these studies. In whole kidney culture, $B m p 7$ mutant kidneys undergo extensive tubulogenesis. This is not the predicted result if BMP7 acts primarily to suppress apoptosis in the nephrogenic mesenchyme (Luo et al. 1995; Dudley and Robertson 1997). Interestingly, treatment of whole kidneys with BMP7 results in a decrease in tubulogenesis in the periphery of the explant. Similarly, mesenchyme adjacent to a BMP7-soaked bead does not express markers of induction following coculture with spinal cord. Collectively, these data demonstrate that BMP7 activity is one component of a pathway controlling the rate of nephrogenesis by repressing tubulogenesis. One possible mechanism to account for the inhibition of tubulogenesis is the down-regulation of genes required for response of the mesenchyme to inductive signals. Currently little is known about the pathway downstream of the inductive signals. Only one gene product, the transcription factor WT1, has been shown to be required cell autonomously for induction of the mesenchyme (Kreidberg et al. 1993). Consistent with this mode of action, our analysis revealed a decrease in expression of WT1 in kidneys treated with BMP7 and FGF2 versus untreated controls (Fig. 6). Further analysis will determine whether this is attributable to changes in the expression level in individual cells or a decrease in the absolute number of expressing cells. Similarly, BMP7 signaling can suppress expression of $B m p 7^{1 a c Z}$, a marker of induced mesenchyme, in explants treated with FGF2 (Fig. 3). Finally, we demonstrate that BMP7 signaling promotes expansion of the stromal progenitor cell population. Implications of this finding are discussed below.
One element common in each of these processes is that BMP7 signaling potentiates the effect of FGF signaling. For example, following pretreatment with both BMP7 and FGF2, explants of metanephric mesenchyme respond more strongly to signals from the spinal cord than cultures treated with FGF2 alone (Fig. 4). These data provide the first demonstration of synergy between BMP and FGF signaling pathways during metanephric development. Similar interactions between the BMP and FGF signaling pathways have also been described in the specification of pre-cardiac mesoderm (Lough et al. 1996). These findings are intriguing because in many other systems, FGF and BMP signaling act antagonistically (Niswander and Martin 1993; Neubuser et al. 1997; Wappner et al. 1997; Ericson et al. 1998; Kretzschmar and Massagué 1998). Such disparate results are not unexpected, however, as both the FGF and TGF $\beta / B M P$ signal transduction pathways are highly complex and sensitive to both extracellular and intracellular environments. Based on current understanding of the FGF and BMP signal transduction pathways, we cannot propose a mechanism governing the interactions we observe in the kidney. In part, this is because of a lack of information concerning the expression of FGF family members in the kidney. Recently, the first defect in kidney development caused by a mutation in an FGF family member (Fgf7) was reported (Qiao et al. 1999). Expression, however, of Fgf7 exclusively in the mature stroma and the limited disruption of nephrogenesis as a result of the mutation suggest that FGF7 signaling does not mediate the events described here. In contrast, BMP7 is expressed in both the ureter and the nephrogenic mesenchyme (Lyons et al. 1995; Dudley and Robertson 1997). In addition, BMP7 is the only BMP family member identified to date that is expressed in the nephrogenic mesenchyme, whereas expression of BMP family members in the stromal progenitor cells has not been described (Lyons et al. 1995; Dudley and Robertson 1997). As it appears to act as a shortrange signal (Fig. 2; Vukicevic et al. 1994), BMP7 produced by the nephrogenic mesenchyme is likely to mediate effects on both cell populations in vivo.

\section{Interactions between stromal progenitor cells and metanephric mesenchyme}

The observation that BMP7 and FGF signaling together result in growth of the stromal progenitor cell population has important implications for tissue interactions during nephrogenesis. The current model of nephrogenesis involves two sets of interactions between three cell populations. First, reciprocal interactions between the ureter and mesenchyme promote growth and branching of the ureter, as well as survival and differentiation of the mesenchyme. Second, the stromal progenitor cells and/ or mature stromal cells signal to the nephrogenic mesenchyme to control nephrogenesis. The latter interaction was first revealed by a disruption of kidney morphogenesis using antibodies directed against GD3 (Sariola et al. 1988), a cell-surface molecule expressed on stromal cells, and later elaborated on through analysis of BF2 
(Hatini et al. 1996) and, more recently, retinoic acid receptor (RAR) mutant embryos (Mendelsohn et al. 1999). The RAR mutations do not affect nephrogenesis significantly, whereas a loss of BF2 function in stromal progenitor cells resulted in a significant reduction in nephrogenesis, apparently without altering the size of the stromal cell population. Here we demonstrate a novel interaction between the nephrogenic mesenchyme and the stromal progenitor cells. In whole kidney cultures, expansion of the stromal progenitor cell population by FGF2 is enhanced by BMP7, a ligand produced by induced nephrogenic mesenchyme (Lyons et al. 1995; Dudley and Robertson 1997; Godin et al. 1998). Therefore the condensed mesenchyme and stromal progenitor cells interact reciprocally and not unidirectionally as suggested previously (Hatini et al. 1996; Mendelsohn et al. 1999). An important implication of this model is that development of the stromal progenitor cell and mature stromal cell populations are controlled throughout kidney development by the induced mesenchyme. These results extend previous studies demonstrating an influence of induction on establishment of stromal/stromal progenitor cell populations in cultures of isolated metanephric mesenchyme (Aufderheide et al. 1987). One caveat to our model is that BMP7 can signal through several different receptors (Yamashita et al. 1995), the expression patterns of which have not been analyzed in detail in the developing kidney. Therefore it is possible that BMP7 signals only to the nephrogenic mesenchyme via an autocrine loop and the interaction we describe is mediated indirectly by BMP7 through an unidentified molecule. One prediction of this model is that loss of BMP7 signaling in the kidney would result in a decrease in stromal progenitor cell formation. In contrast, kidneys from $B m p 7$ null embryos appear to contain a normal stromal progenitor cell population by virtue of BF2 expression (A.T. Dudley, unpubl.). This does not, however, rule out a role for BMP7 signaling in the development or function of stromal progenitor cells. Because FGF signaling alone can stimulate growth of the stromal progenitor cell population (Fig. 5), these data only suggest that BMP7 is not a limiting factor in this process.

An interesting possibility suggested by these data is that signals from stromal progenitor cells may have an important role in maintaining a population of nephrogenic mesenchyme at the tips of the branching ureter. We infer this from several observations. FGF2 signaling maintains nephrogenic mesenchyme cells in the absence of spinal cord (Fig. 4) and promotes expansion of the stromal progenitor cell population (Figs. 6 and 7). In contrast, BMP7 signaling alone neither maintains competent nephrogenic mesenchyme in culture nor results in growth of the stromal progenitor cell population in intact kidney explants. Therefore, it is possible that signals from the stromal progenitor cells help maintain the nephrogenic mesenchyme cell population (Fig. 4). If this is correct, the increased response to spinal cord of mesenchyme explants treated with both FGF2 and BMP7 (Fig. 4) may be attributable to an increase in size of (Figs.
3 and 6) or signaling by the stromal progenitor cell population. In addition, a correlation between the increase in size of the stromal progenitor cell population and decrease in nephron formation in kidney explants (Fig. 6 and 7) suggests that signals from stromal progenitor cells may also maintain nephrogenic mesenchyme by antagonizing tubulogenesis. This assertion, however, contradicts the conclusions of Hatini et al. (1996). Their analysis of BF2 mutant kidneys suggests that signaling by stromal progenitor cells promotes nephron formation. This paradox can be resolved if BF2 function does not promote tubulogenesis but is required to negatively regulate a stromal progenitor cell signaling pathway that antagonizes tubulogenesis. In this case, a loss of BF2 would result in an increase in signals opposing differentiation. If nephrogenic mesenchyme cells continue to proliferate without differentiating into nephrons, the condensates of mesenchyme surrounding the tips of the ureteric branches will expand. Therefore, this model can account for the primary defects observed during development of BF2 mutant kidneys (Hatini et al. 1996). If this prediction is correct, the decrease in tubulogenesis observed in BF2 mutant kidneys might be partially compensated for by the increased propensity of $B m p 7$ mutant mesenchyme to differentiate (Fig. 5).

In summary, these data suggest several important modifications to current models of nephrogenesis (Fig. 8). Following contact, reciprocal interactions between the ureteric epithelium and metanephric blastema result in the establishment and maintenance of BMP7 production by the nephrogenic mesenchyme cell population. For the purpose of this model, we presume that nephrogenic mesenchyme cells are the source of BMP7 regulating the processes described here because of the apparent short-range effects of BMP7 in culture. We do not, however, preclude the possibility that BMP7 produced by the ureter has an important role. Adjacent to the tips of the ureter, autocrine BMP7 signaling combined with signaling through FGFR2 maintains a population of nephrogenic mesenchyme cells at the tips of the branching ureter by repressing apoptosis and opposing differentiation of the mesenchyme. In addition, paracrine signaling by BMP7 interacts with FGF signaling through FGFR1 to promote proliferation of the BF2-positive stromal progenitor cells, which in turn provide signals that help maintain the nephrogenic mesenchyme cell population. Although in our model differentiation of the nephrogenic mesenchyme is repressed by both signals from the stromal progenitor cells and by autocrine BMP7 signaling, these two pathways may not function equally in vivo. Exogenously added BMP7 and FGF2 increases signaling in metanephric mesenchyme cell populations leading to growth of the stromal progenitor cells and a decrease in tubulogenesis. Based on this model of nephrogenesis, the defects observed in Bmp7 mutant kidneys may be attributable directly to a failure of BMP7 signaling in the nephrogenic mesenchyme or, indirectly, to a loss of signals from the stromal progenitor cells. 


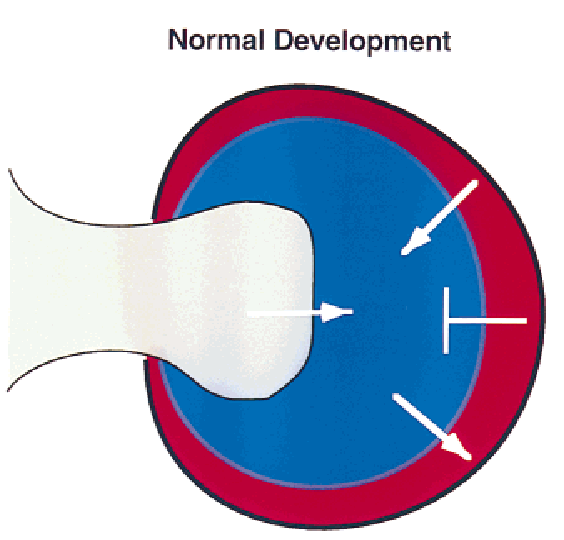

Stromal Precursor cells BF-2 FGFR1

Nephrogenic Mesenchyme BMP7 WT-1

Ureter BMP7 Pax-2

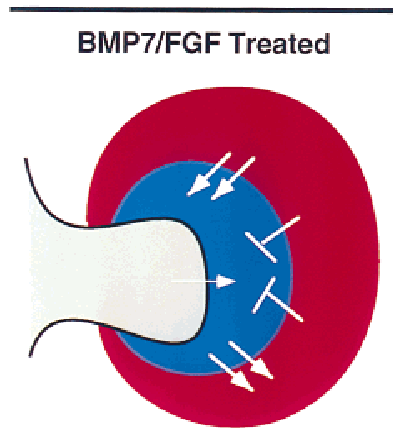

BMP7 Mutant

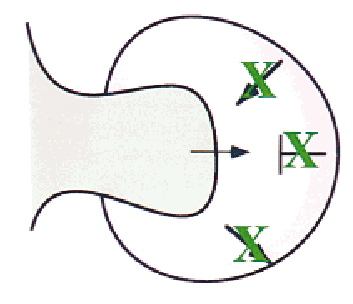

Figure 8. Model of kidney development. This model represents a ureteric bud that has just entered the metanephric mesenchyme and displays the signaling that occurs between the various cell types of the developing kidney based on data in the literature and results presented here. During normal development, signals from the ureteric bud (gray) are required for induction of the nephrogenic mesenchyme (blue). In turn, these cells signal to the adjacent stromal progenitor cells (red) promoting their survival, growth, and differentiation. In a reciprocal manner, the stromal progenitor cells send an antidifferentiation signal to the nephrogenic mesenchyme to control the rate of nephrogenesis. In addition, these cells may also send a survival factor to the nephrogenic mesenchyme. Potential molecules involved in these signaling events are listed. In BMP7/ FGF2-treated kidney explants, increased levels of BMP7 and FGF lead to increased survival of the nephrogenic mesenchyme as well as to an expansion of the stromal precursor cells. As a result, there is an increased level of the antidifferentiation signal coming from the stromal precursor cells. Therefore, differentiation of the nephrogenic mesenchyme is reduced. At this time, the source of the FGF signal is unknown. In $B m p 7$ mutant kidneys, lack of BMP7 signaling (white) leads to loss of proper signaling from the stromal precursor cells (pink), thereby resulting in premature differentiation and perhaps increased cell death in the nephrogenic mesenchyme.

\section{Materials and methods}

\section{Mouse lines}

Derivation, maintenance and genotyping of the $B m p 7^{m 1 R o b}$ and the $B m p 7^{\text {lac } Z}$ mouse lines were described previously (Dudley et al. 1995; Godin et al. 1998). ICR strain of mice were obtained from Taconic.

\section{Tissue culture}

Explant cultures Animals were sacrificed by asphyxiation with halothane. Kidneys were isolated from 11.5-dpc embryos and dissected in L-15 medium supplemented with $1 \%$ serum. To obtain isolated mesenchyme, kidneys were treated with $0.25 \%$ trypsin for $6 \mathrm{~min}$ on ice, then washed in dissection medium (L-15 medium supplemented with $1 \%$ serum). The mesenchyme was teased away from the ureter using 26G needles. Explants of mesenchyme or whole kidneys were grown at $37^{\circ} \mathrm{C}$, $5 \% \mathrm{CO}_{2}$ on $0.1 \mu \mathrm{m}$ pore size Nuclepore polycarbonate filters (Corning) in F12/DMEM 1:1 (GIBCO) supplemented with either $10 \%$ defined fetal bovine serum (Hyclone) or $50 \mu \mathrm{g} / \mathrm{ml}$ of human transferrin (GIBCO) and 1\% insulin/transferrin/selenium (Sigma). For transfilter cultures, the spinal cord was cultured between two 1- $\mu \mathrm{m}$ filters on a Trowell-type screen. Freshly isolated mesenchyme was then placed on top. In some experiments, the medium was supplemented with $100 \mathrm{ng} / \mathrm{ml}$ FGF2 (Sigma, catalog no. F-0291). At least three explants per treatment were prepared for each experiment. Experiments were repeated three to six times on different days. Recombinant BMP7 was obtained from Creative Biomolecules (Framingham, MA). Activity of stock solutions was tested by the method of Ruppert et al. (1996). Affigel blue beads were prepared as described previously (Godin et al. 1998).

$X$-gal staining for lacZ activity Kidney explants were fixed in $\mathrm{X}$-gal buffer (PBS, $5 \mathrm{~mm}$ EGTA, $2 \mathrm{~mm} \mathrm{MgCl} \cdot 6 \mathrm{H}_{2} \mathrm{O}, 0.2 \% \mathrm{NP}-40$, $0.2 \mathrm{~mm}$ deoxycholate) containing $1 \%$ formaldehyde and $0.2 \%$ glutaraldehyde and then washed three times in X-gal buffer. Explants were stained overnight at $37^{\circ} \mathrm{C}$ in X-gal buffer containing $5 \mathrm{~mm}$ each potassium ferricyanide/potassium ferrocyanide, $0.5 \mathrm{mg} / \mathrm{ml} \mathrm{X}$-gal, followed by three washes in X-gal wash buffer, and post-fixed in $4 \%$ paraformaldehyde for $2 \mathrm{hr}$. Kidney explants were again washed three times in X-gal buffer and then either stored as whole mounts or processed for sectioning.

Detecting cell death Cultures were incubated in a $2 \%$ solution of TOPRO-1 (Molecular Probes) in Ham's F12 (Sigma) for $30 \mathrm{~min}$ at $37^{\circ} \mathrm{C}$. Labeled tissues were then washed in large volumes of Ham's F12 at $37^{\circ} \mathrm{C}$ for $15-30$ min. Samples were mounted on slides in Ham's F12, and labeled cells were detected using a fluorescein filter set on a Leitz DMR microscope.

DiI labeling DiI (Molecular Probes) was dissolved in ethanol according to the manufacturer's instructions. A glass microinjection pipette was used to place a small amount of dye into the explant. Explants were cultured for an additional 2 days. Fluorescent signal was detected using a rhodamine filter set.

\section{In situ hybridization}

Histology Explants on filters were fixed in 4\% paraformaldehyde/PBS or Bouin's fixative at $4^{\circ} \mathrm{C}$ overnight, followed by dehydration through a graded ethanol series. The material was cleared in xylenes or n-butanol and embedded in Fibrowax (BDH). Samples were sectioned at $5 \mu \mathrm{m}$ and sections collected on Tespa-treated glass slides. Sections for histology were stained with hematoxylin and eosin using standard procedures.

In situ hybridization Section in situ hybridization (Jones et al. 1991) and whole-mount in situ hybridization (Wall and Hogan 1995) were performed as described. Probes specific for Pax2 (Dressler et al. 1990), Wnt4 (Stark et al. 1994), WT1 (Kreidberg et al. 1993), BF2 (Hatini et al. 1996), Bmp7 (Dudley and Robertson 1997), and Fgfr1 and Fgfr2 (Orr-Urtreger et al. 1991) were used as 
described. Sections were photographed using a Leitz DMR microscope and Fujichrome Velvia color slide film or TechPan black and white film.

Immunohistochemistry For analysis of epithelial development, whole kidneys or explants were fixed in methanol at $-20^{\circ} \mathrm{C}$ then rehydrated in PBS $+0.1 \%$ Tween 20 (PBT). Samples were blocked in $10 \%$ sheep serum/PBT for $1 \mathrm{hr}$ then incubated in 1:400 rabbit anti-mouse laminin antibody (Sigma) in 1\% serum/PBT overnight at $4^{\circ} \mathrm{C}$ with rocking. The next day, samples were washed extensively with PBT followed by an overnight wash at $4^{\circ} \mathrm{C}$. The primary antibody was detected using a Texas Red-conjugated goat anti-rabbit secondary antibody at 1:400 in PBT for $4 \mathrm{hr}$. Samples were washed extensively at room temperature then overnight at $4^{\circ} \mathrm{C}$. Cultures were mounted in $90 \%$ glycerol containing $1 \mathrm{mg} / \mathrm{ml}$ p-phenylenediamine (Sigma). Staining was visualized using a rhodamine filter set on a Leitz DMR microscope. Images were captured on Ektachrome Elite II 400 ASA film.

\section{Acknowledgments}

We thank T. Kuber Sampath for generously supplying recombinant protein; Kimberly Tremblay for critical reading of the manuscript; members of the Robertson laboratory for useful discussions; and Patti Lewko and Joe Rocca for expert animal care. This work was supported by grants to E.J.R. from the National Institutes of Health (NIH). R.E.G. was supported by a postdoctoral fellowship from the NIH.

The publication costs of this article were defrayed in part by payment of page charges. This article must therefore be hereby marked 'advertisement' in accordance with 18 USC section 1734 solely to indicate this fact.

\section{References}

Aufderheide, E., R. Chiquet-Ehrismann, and P. Ekblom. 1987. Epithelial-mesenchymal interactions in the developing kidney lead to expression of tenascin in the mesenchyme. J. Cell Biol. 105: 599-608.

Barasch, J., J. Qiao, G. McWilliams, D. Chen, J.A. Oliver, and D. Herzlinger. 1997. Ureteric bud cells secrete multiple factors, including bFGF, which rescue renal progenitors from apoptosis. Am. J. Physiol. 273 F757-767.

Cancilla, B., J. Cauchi, B. Key, V. Nurcombe, D. Alcorn, and J. Bertram. 1996. Immunolocalization of fibroblast growth factor-1 and -2 in the embryonic rat kidney. Nephrology 2: 167174.

Coles, H.S.R., J.F. Burne, and M.C. Raff. 1993. Large-scale normal cell death in developing rat kidney and its reduction by epidermal growth factor. Development 118: 969-981.

Davies, J.A. and D.R. Garrod. 1995. Induction of early stages of kidney tubule differentiation by lithium ions. Dev. Biol. 167: 50-60.

Dressler, G.R., U. Deutsch, K. Chowdhury, H.O. Nornes, and P. Gruss. 1990. Pax2, a new murine paired-box containing gene and its expression in the developing excretory system. Development 109: 787-795.

Dudley, A., K. Lyons, and E.J. Robertson. 1995. A requirement for bone morphogenetic protein-7 during development of the mammalian kidney and eye. Genes \& Dev. 9: 2795-2807.

Dudley, A.T. and E.J. Robertson. 1997. Overlapping expression domains of bone morphogenetic protein family members potentially account for limited tissue defects in BMP7 deficient embryos. Dev. Dynamics 208: 349-362.
Ericson, J., S. Norlin, T.M. Jessell, and T. Edlund. 1998. Integrated FGF and BMP signaling controls the progression of progenitor cell differentiation and the emergence of pattern in the embryonic anterior pituitary. Development 125: $1005-1015$.

Godin, R.E., N. Takaesu, E.J. Robertson, and A.T. Dudley. 1998. Regulation of Bmp7 expression during kidney development. Development 125: 3473-3482.

Gossens, C.L. and B.R. Unsworth. 1972. Evidence for a two-step mechanism operating during in vitro mouse kidney tubulogenesis. J. Embryol. Exp. Morphol. 28: 615-631.

Gurdon, J.B. 1988. A community effect in animal development. Nature 336: 772-774.

Hatini, V., S.O. Huh, D. Herzlinger, V.C. Soares, and E. Lai. 1996. Essential role of stromal mesenchyme in kidney morphogenesis revealed by targeted disruption of a winged-helix transcription factor BF2. Genes \& Dev. 10: 1467-1478.

Jones, C.M., K.M. Lyons, and B.L.M. Hogan. 1991. Involvement of bone morphogenetic protein-4 (BMP-4) and Vgr-1 in morphogenesis and neurogenesis in the mouse. Development 111: $531-542$

Koseki, C., D. Herzlinger, and Q. Al-Awqati. 1992. Apoptosis in metanephric development. J. Cell Biol. 119: 1327-1333.

Kreidberg, J.A., H. Sariola, J.M. Loring, M. Maeda, J. Pelletier, D. Housman, and R. Jaenisch. 1993. WT1 is required for early kidney development. Cell 74: 679-691.

Kretzschmar, M. and J. Massagué. 1998. SMADs: Mediators and regulators of TGF-beta signaling. Curr. Opin. Gene. \& Dev. 8: 103-111.

Lough, J., M. Barron, M. Brogley, Y. Sugi, D.L. Bolender, and X. Zhu. 1996. Combined BMP-2 and FGF-4, but neither factor alone, induces cardiogenesis in non-precardiac embryonic mesoderm. Dev. Biol. 178: 198-202.

Luo, G., C. Hofmann, A.L. Bronckers, M. Sohocki, A. Bradley. and G. Karsenty. 1995. BMP7 is an inducer of nephrogenesis, and is also required for eye development and skeletal patterning. Genes \& Dev. 9: 2808-2820.

Lyons, K.M., B.L.M. Hogan, and E.J. Robertson. 1995. Colocalization of BMP7 and BMP-2 RNAs suggests that these factors cooperatively mediate tissue interactions during murine development. Mech. Dev. 50 71-83.

Mendelsohn, C., E. Batourina, S. Fung, T. Gilbert, and J. Dodd. 1999. Stromal cells mediate retinoid-dependent functions essential for renal development. Development 126: 11391148.

Miyamoto, N., M. Yoshida, S. Kuratani, I. Matsuo, and S. Aizawa. 1997. Defects of urogenital development in mice lacking Emx2. Development 124: 1653-1664.

Neubuser, A., H. Peters, R. Balling, and G.R. Martin. 1997. Antagonistic interactions between FGF and BMP signaling pathways: A mechanism for positioning the sites of tooth formation. Cell 90: 247-255.

Niswander, L. and G.R. Martin. 1993. FGF-4 and BMP-2 have opposite effects on limb growth. Nature 361: 68-71.

Orr-Urtreger, A., D. Givol, A. Yayon, Y. Yarden, and P. Lonai. 1991. Developmental expression of two murine fibroblast growth factor receptors, flg and bek. Development 113: $1419-1434$.

Perantoni, A.O., L. Dove, and C. Williams. 1991. Induction of tubules in rat metanephric mesenchyme in the absence of an inductive tissue. Differentiation 48: 25-31.

Perantoni, A.O., L. Dove, and I. Karavanova. 1995a. Basic fibroblast growth factor can mediate the early inductive events in renal development. Proc. Natl. Acad. Sci. 92: 4696-4700.

. 1995b. Growth and branching morphogenesis of rat collecting duct anlagen in the absence of metanephrogenic mes- 
enchyme. Differentiation 48: 107-113.

Qiao, J., R. Uzzo, T. Obara-Ishihara, L. Degenstein, E. Fuchs, and D. Herzlinger. 1999. FGF-7 modulates ureteric bud growth and nephron number in the developing kidney. Development 126: 547-554.

Ruppert, R., E. Hoffmann, and W. Sebald. 1996. Human bone morphogenetic protein 2 contains a heparin binding site which modifies its biological activity. Eur. J. Biochem. 237: 295-302.

Sainio, K., D. Nonclercq, M. Saarma, J. Palgi, L. Saxen, and H. Sariola. 1994. Neuronal characteristics in embryonic renal stroma. Int. J. Dev. Biol. 38: 77-84.

Sariola, H., E. Auferheide, H. Bernhard, S. Henke-Fahle, W. Dippold, and P. Ekblom. 1988. Antibodies to cell surface ganglioside GD3 perturb inductive epithelial-mesenchymal interactions. Cell 54: 235-245.

Saxen, L. 1987. Organogenesis of the kidney (ed. P.W. Barlow, P.B. Green, and C.C. White), Vol. 19, Cambridge University Press, Cambridge, UK.

Shah, N.M., A.L. Groves, and D.J. Anderson. 1996. Alternative neural crest cell fates are instructively promoted by TGFbeta superfamily members. Cell 85: 331-344.

Stark, K.L., J.A. McMahon, and A.P. McMahon. 1991. FGFR-4, a new member of the fibroblast growth factor receptor family, expressed in the definitive endoderm and skeletal muscle lineages of the mouse. Development 113: 641-651.

Stark, K., S. Vainio, G. Vassileva, and A.P. McMahon. 1994. Epithelial transformation of metanephric mesenchyme in the developing kidney regulated by Wnt4. Nature 372: 679683.

Varley, J.E., R.G. Wehby, D.C. Rueger, and G.D. Maxwell. 1995. Number of adrenergic and islet-1 immunoreactive cells is increased in avian trunk neural crest cultures in the presence of human recombinant osteogenic protein-1. Dev. Dyn. 203: 434-447.

Vukicevic, S., V. Latin, P. Chen, R. Batorsky, A.H. Reddi, and T.K. Sampath. 1994. Localization of osteogenic protein-1 (bone morphogenetic protein-7) during human embryonic development: High affinity binding to basement membranes. Biochem. Biophys. Res. Commun. 198: 693-700.

Vukicevic, S., J.B. Kopp, F.P. Luyten, and T.K. Sampath. 1996. Induction of nephrogenic mesenchyme by osteogenic protein 1 (bone morphogenetic protein 7). Proc. Natl. Acad. Sci. 93: 9021-9026.

Wall, N.A. and B.L.M. Hogan. 1995. Expression of bone morphogenetic protein-4 (BMP-4), bone morphogenetic protein-7 (BMP7), fibroblast growth factor-8 (FGF-8) and sonic hedgehog $(\mathrm{SHH})$ during branchial arch development in the chick. Mech. Dev. 53: 383-392.

Wappner, P., L. Gabay, and B.-Z. Shilo. 1997. Interactions between the EGF receptor and DPP pathways establish distinct cell fates in the tracheal placodes. Development 124: 47074716.

Weller, A., L. Sorokin, E.M. Illgren, and P. Ekblom. 1991. Development and growth of mouse embryonic kidney in organ culture and modulation of development by soluble growth factors. Dev. Biol. 144: 248-261.

Yamashita, H., P. ten Dijke, D. Huylebrowck, T.K. Sampath, M. Andries, J.C. Smith, C.-H. Heldin, and K. Miyazono. 1995. Osteogenic protein-1 binds to activin type II receptors and induces certain activin-like effects. J. Cell Biol. 130: $217-$ 226.

Zhou, M., R.L. Sutliff, R.J. Paul, J.N. Lorenz, J.B. Hoying, C.C. Haudenschild, M. Yin, J.D. Coffin, L. Kong, E.G. Kranias et al. 1998. Fibroblast growth factor 2 control of vascular tone. Nat. Med. 4: 201-207. 


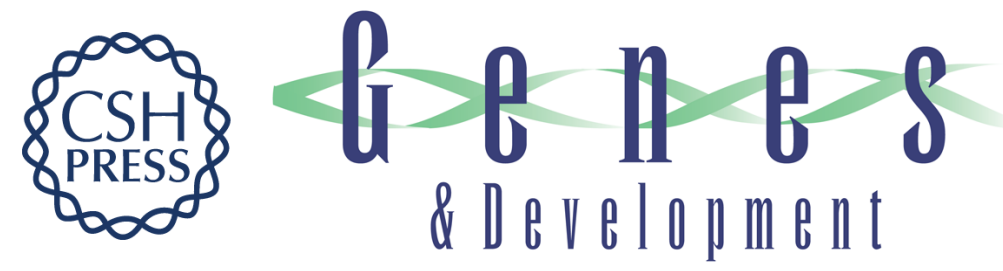

\section{Interaction between FGF and BMP signaling pathways regulates development of metanephric mesenchyme}

Andrew T. Dudley, Robert E. Godin and Elizabeth J. Robertson

Genes Dev. 1999, 13:

\section{License}

Email Alerting Receive free email alerts when new articles cite this article - sign up in the box at the top Service right corner of the article or click here.

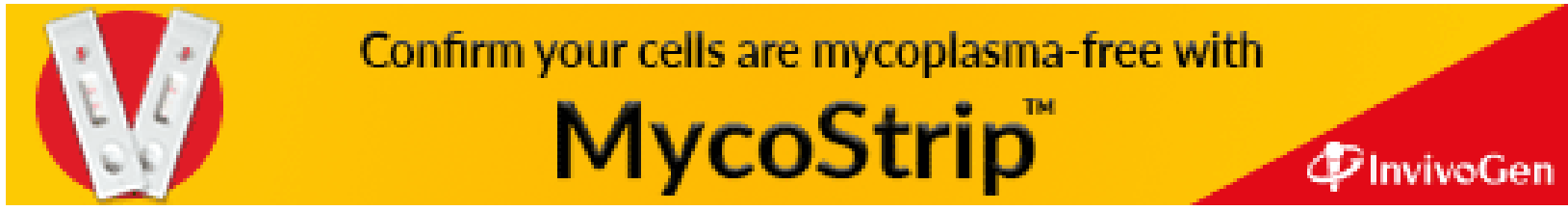

